\title{
Claves para repensar el mundo circundante. Una expansión de la noción de Werkwelt de M. Heidegger por medio del concepto de "conjunto técnico" de G. Simondon
}

\author{
Keys to re-think the Sorrounding World: \\ An expansion of Heidegger's notion of Werkwelt through G. \\ Simondon's concept of Technical Conjunct
}

Luciano Mascarón

Resumen: Este trabajo se propone mostrar que la noción de mundo circundante técnico [Werkwelt elaborada por M. Heidegger no logra dar cuenta de la complejidad de la realidad técnica actual puesto que se basa en un modelo artesanal y cuasi-privado de producción. Sugerimos que, de ser consecuentes con las propuestas del mismo Heidegger, y descubrir en el medio técnico a la naturaleza (materia, energía y causalidades) y al mundo público, el Werkwelt debería expandirse hasta coincidir con un entramado pluri-sistémico en una soldadura de zonas funcionales de muy diversa índole, es decir, un conjunto técnico, noción elaborada por G. Simondon

Palabras clave: Técnica, Mundo del trabajo, Simondon, Heidegger, Conjunto técnico

Abstract: This paper attempts to show that Heidegger's notion of Technical Sorrounding World [Werkwelt] does not account for the complexity of today's technical reality, since it is based upon a quasi-private and artisanal model of production. We suggest that, if we are to be consequent with Heidegger's own proposals, and discover nature (as matter, energy and causalities) and the public world within the technical enviroment, the Werkwelt should be expanded until it matches a multi-sistemic

\footnotetext{
* Doctor en Filosofía por la Universidad de Buenos Aires. Investigador Asistente en el Consejo Nacional de Investigaciones Científicas y Técnicas (CONICET). Profesor adjunto en la materia "Filosofía del Lenguaje" en la Carrera de Filosofía de la Universidad Católica Argentina (UCA). Profesor adjunto en la materia "Filosofía de la Naturaleza" en la Carrera de Filosofía de la Universidad del Salvador (USAL). Profesor adjunto en la materia "Metafísica", en la carrera de filosofía de la universidad de ciencias empresariales y sociales (UCES). Dirección electrónica: lcnmascaro@hotmail.com
} 
network, in a welding of functional zones of a very diverse fashion, that is, a Technical Conjunct, a notion coined by G. Simondon

Keywords: Technology, World of Work, Simondon, Heidegger, Technical Conjunct

\section{Sobre la delimitación de las obras analizadas}

En primer lugar, es necesario realizar una precisa delimitación del período a estudiar. En efecto, en este análisis nos centraremos respecto de Heidegger en las obras del así llamado "período de Marburgo", delimitación que responde a una serie de motivos que a continuación explicamos.

El período de Marburgo (1923-1929) es frecuentemente relegado en los análisis de la posición de Heidegger acerca de la técnica. Esta relativa falta de interés en el período puede responder al hecho de que los años anteriores y posteriores a los '20 resultan mucho más seductores a la hora de estudiar cuestiones relacionadas con el tema. Específicamente, las obras tempranas de Heidegger realizan un minucioso análisis fenomenológico de la filosofía aristotélica, donde la téchne ocupa un lugar central. Por su parte, las obras posteriores a los años '30 parecen volcarse de manera más específica al problema; prueba de esta aparente concentración en la temática es la aparición de conferencias cuyo título se menciona expresamente la cuestión del objeto técnico (pensemos en "la pregunta por la técnica", "la cosa", "lenguaje tradicional y lenguaje técnico" o "construir, habitar, pensar").

La suspensión del análisis de los textos posteriores a los '30 puede parecer, por tanto, sorprendente: ¿no se dedica allí nuestro autor, específicamente, a interpretar la estructura funcional interna de los grandes sistemas industriales de extracción de energía? Consideramos que la respuesta es negativa. El segundo Heidegger sólo menciona una serie de complejos técnicos, pero no analiza los verdaderos procesos que ocurren en su interior, como si fuese de común conocimiento, por ejemplo, el funcionamiento de una planta nuclear. El interés del segundo Heidegger no está dirigido a la elucidación de la estructura y de los encadenamientos físicos, causales y operativos de los entes técnicos o de los sistemas industriales, sino, según él mismo lo indica, al descubrimiento de la esencia de la técnica, y dado que "la 
esencia de la técnica no es nada técnico"1 y que la concentración reflexiva en los artefactos, máquinas y sistemas constituirían un desvío o resignación ante la urgente cuestión de la determinación de la esencia de la técnica, difícilmente encontraremos en su pensamiento posterior a la Kehre una descripción del modo en que los complejos industriales llegan a desdibujar los límites del mundo circundante. El segundo Heidegger suspende el análisis óntico de los objetos técnicos, es por ello que no podremos seguirlo para aportar claridad a la cuestión que nos interesa. La separación de la temática de nuestro interés es expresada por el segundo Heidegger en estos términos: "[si la esencia de la técnica fuese algo técnico], la turbina de vapor, la emisora de radio, el ciclotrón serían una estructura de emplazamiento [Gestell. Pero la palabra Gestell no mienta ahora ningún aparato, ningún tipo de maquinaria". ${ }^{2}$

El Heidegger de la Kebre desestima demasiado rápidamente el estudio pormenorizado de los sistemas técnicos por considerarlos representantes de algo más esencial: la estructura de emplazamiento como carácter definitorio de la actitud provocadora humana que descubre al mundo en tanto stock y reserva de energías permanentemente disponibles. Semejante decisión interpretativa no será capaz de descubrir, por ejemplo, los límites naturales que las mismas causalidades físicas traen a la extracción de energía. Sin la revisión que se interna en las fábricas y plantas energéticas, no se verá la limitación de las fuerzas dominadoras del ser humano, y la técnica aparecerá como una fuerza universalmente desplegada y amenazante.

Si bien es cierto que en escritos tales como "La cosa", "Construir, habitar, pensar", o incluso "El origen de la obra de arte", Heidegger analiza artefactos en un intento por mostrar que el ente aporta a la experiencia significativa del mundo una cierta opacidad interpretativa, o bien una materialidad ("tierra") que escapa al eje "antropológico" de configuración de sentido (una característica de los entes técnicos que nosotros intentaremos resaltar en este trabajo), se observa, al mismo tiempo, que el útil siempre es presentado como momento de manifestación de algo más originario (el ereignis, la cuadratura, la verdad del Ser [Seyn], la Gestell, etc.). Así, en los escritos y

${ }^{1}$ Heidegger, Martin, "La pregunta por la técnica”, en Conferencias y artículos, trad. de Eustaquio Barjau, Barcelona, Ediciones de Serbal, 1994, p. 9.

2 Ibidem, p. 32. 
conferencias posteriores a los años '30 los objetos técnicos dejan de ser abordados con interés filosófico-tecnológico. En su lugar, se accede a ellos con la intención de superarlos en vistas a una elucidación ontológica del sentido del ser y el mundo.

Asimismo, a partir de la Kehre el modelo entitativo con el que se estudian los entes se modifica. Ya no se seguirá el paradigma del útil (como en el período de Marburgo) sino el de la obra de arte: un ente caracterizado por una fase de transparencia (puesto que coincide con los entes técnicos en el hecho de haber sido producido), pero también por una fase de opacidad semántica (puesto que la finalidad como clave de interpretación y sentido queda cancelada en la independencia referencial que la obra funda). Los análisis de artefactos que se realicen a partir del modelo del objeto artístico (el puente, la jarra, la copa de plata, etc.) no podrán evitar ofrecer versiones "estetizadas" de los objetos técnicos; en otras palabras, el útil deja de ser pensado desde él mismo. Los artefactos ya no se estudian con interés técnico, el interés de Heidegger es la ontología, y no precisamente la ontología de los entes técnicos. Algo incluso más radical ocurre en los escritos más tardíos de Heidegger, que, como el propio autor indica en su autointerpretación de la Kehre, siguen el objetivo de "pensar el ser sin el ente". 3

En definitiva, nuestra decisión interpretativa busca resaltar un período que, por los motivos expuestos, es frecuentemente olvidado en el estudio de estas temáticas.

\section{Introducción}

El mundo circundante [Umwelt] es una modalidad del mundo en tanto carácter existencial del Dasein. El Umwelt es el mundo tal como es descubierto por la mirada circunspectiva del trato pragmático con los entes. Heidegger indica que existen dos modalidades principales del mundo circundante: el

${ }^{3}$ Esta expresión aparece, por ejemplo, en Heidegger, Martin, Zur sache des Denkens (edición de F.-W. von Herrmann, Gesamtausgabe, Frankfurt am Main, Vittorio Klostermann, vol. 14, 2007, pp. 51-52). 
mundo de la naturaleza [Welt der Natur], y el mundo del trabajo [Werkwelt], ${ }^{4}$ es decir, el medio donde se despliega la ocupación técnica. Al analizar la noción de Werkwelt, Heidegger indica que, en medio del trato ocupacional con entes técnicos hacen su aparición (aunque de modo inexplícito) dos factores de gran relevancia: en primer lugar, la naturaleza (en una serie de sentidos que estudiaremos oportunamente) y en segundo lugar, los otros (esto es, las otras existencias humanas), que convierten a ese mundo de la ocupación en un mundo público repleto de usuarios y productores, un mundo donde acontece un diseño de artefactos que tiene en cuenta su utilizabilidad por todos los seres humanos en general, y por ninguno en particular.

Ahora bien, al exponer la noción de mundo público, Heidegger se dedica casi exclusivamente a analizar el fenómeno de la caída, esto significa que la noción de mundo público aparece íntimamente asociada a la noción de Uno [Das Man] y de estado público de interpretación. Si bien sabemos que el mundo público está poblado por otros seres humanos, y comprendemos que todos ellos se comportan técnicamente en relación con el mundo, no encontramos en el primer período del pensar de Heidegger un análisis pormenorizado de aquellas tareas y relaciones humanas que constituyen, precisamente, el carácter público del mundo común; en otras palabras, Heidegger no explicita lo que pueda significar ese mundo público en sentido técnico ${ }^{5}$ ni de qué manera la consideración de los otros podría llegar a alterar la noción de mundo del trabajo.

En este estudio nos dedicaremos específicamente a la consideración del mundo circundante en tanto mundo circundante técnico o Werkwelt. En este sentido, procuraremos extraer las consecuencias de la afirmación heideggeriana de que en el mundo de la ocupación hacen su aparición la naturaleza y lo público, para sugerir una expansión de la noción de mundo circundante técnico en el concepto más contemporáneo de conjunto técnico de G. Simondon. En nuestro camino descubriremos la insuficiencia del sistema artesanal de producción como modelo de la actividad técnica humana, y la insuficiencia del espacio técnico del taller como modelo para espacios técnicos más contemporáneos donde se

4 Heidegger, M., Prolegómenos para una bistoria del concepto de tiempo, trad. de Jaime Aspiunza, Madrid, Alianza, 2007, § 23b- $\alpha$.

5 Aunque sí estudia profundamente la relación del mundo público con los fenómenos de la caida y la impropiedad. 
verifica un complejo entrelazamiento de sistemas, procesos y actividades humanas.

\section{Primera parte: mundo circundante, mundo público y naturaleza.}

La noción existencial de mundo lo define como un entramado de relaciones significativas, una red de sentidos interdependientes en la cual cada ente o núcleo significativo dice referencia a los demás, y obtiene de ellos su sentido, en función del proyectarse fáctico de una existencia. El mundo de la cotidianidad media encuentra un tipo de ente paradigmático: el útil o artefacto [Zeug]. El uso de artefactos constituye la forma primaria de relación del ser humano con el mundo; para esta modalidad, el artefacto se define por su remisionalidad, todo artefacto "es para" [Um-zu] y en esta finalidad, en este quedar referido al fin práctico, radica su esencia.

La aproximación que destaca el papel fundamental de la existencia en el circuito de sentido de toda ocupación con útiles convierte a la remisionalidad del artefacto en condición respectiva [Bewandtnis]. En efecto, el entramado de remisiones obtiene su sentido de una serie de referencias interrelacionadas: martillo $\rightarrow$ martillar $\rightarrow$ reparar un tejado $\rightarrow$ evitar filtraciones de agua $\rightarrow$ permitir el habitar del Dasein. Como vemos, existe una remisión final de todas las condiciones respectivas: el denominado por mor de qué [Worumwillen o Umwillen], que constituye el centro desde donde brota el sentido de la totalidad respeccional [Bewandtnisganzheit]. "Aquello hacia lo cual algo es dejado respeccionalmente en libertad es una totalidad respeccional, y últimamente, el Mundo, el cual ya debe estar pre-descubierto". ${ }^{6}$ La W orumwillen es la condición de posibilidad para que lo intramundano aparezca como a la mano. ${ }^{7} \mathrm{El}$ mundo, en tanto entramado de entes y finalidades, articuladas por relaciones de significado que se abren en función del proyectar del Dasein y que lo poseen a él como remisión final, queda caracterizado como significatividad [Bedeutsamkeit].

${ }^{6}$ Tugendhat, E., Der Warheitsbegriff bei Husserl und Heidegger, Berlin, Walter de Gruyter \& Co., 1970, p. 290.

${ }^{7}$ Ibidem, p. 288. 
Heidegger aclara que la pertenencia de un útil a una totalidad no es captada de manera explícita. No es necesario, por ejemplo, que el contexto ocupacional del taller sea captado patentemente para habilitar al carpintero a concluir su construcción de la silla (de hecho, la percatación explícita de todos los momentos nodales del plexo de remisiones sería un auténtico obstáculo al quehacer) El triunfo de un artefacto depende en parte de su no-llamatividad, es decir, la ocupación no debe detenerse en la contemplación del útil, sino atravesarlo en dirección a la tarea que debe ser realizada. Heidegger indica que la ocupación se desarrolla dentro de una comprensión inexplícita del mundo, la así llamada comprensión primaria. ${ }^{8} \mathrm{El}$ ser en respectividad constituye ontológicamente al útil, por ello no puede hablarse de un artefacto aislado de un entramado significativo. En cada útil late como trasfondo una totalidad respeccional, caracterizada por su significatividad.

En la obra Prolegómenos para una historia del concepto de tiempo se establece la mencionada distinción entre dos modalidades del mundo circundante: el "mundo del trabajo" [Werkwelt] y el "mundo de la naturaleza" [Welt der Natur]. El mundo del trabajo nombra a aquel ámbito al que la ocupación se abandona sin percibirlo temáticamente, se trata del horizonte en el que se despliegan las labores productivas del Dasein: el mundo como estructura reticular de sentidos pragmáticos interrelacionados, abiertos por el proyecto de la existencia. El mundo del trabajo confirma que el útil nunca comparece de manera aislada, siempre se encuentra en un contexto práctico: la totalidad de útiles [Zeuggan₹̧eit]. En el comparecer del útil ya siempre ha venido a la presencia el taller, el estudio, el aula.

En cuanto a la segunda modalidad, el mundo de la naturaleza -en tanto mundo compuesto de "cosas naturales"-, sólo es posible en un proceso de derivación; su emerger se funda en el previo mundo del trabajo. El mundo de la naturaleza nombra al mundo tal como comparece ante la percepción pura, ${ }^{9}$ un tipo de visión que responde al puro aspecto de lo compareciente, y que requiere, para su acceso a este ámbito, de la previa obturación de la mirada ocupacional. La naturaleza en este sentido aparece como un derivado del

\footnotetext{
${ }^{8}$ Heidegger, M., Lógica: la pregunta por la verdad, trad. de J. Alberto Ciria, Madrid, Alianza, 2004, ऽ $12 \mathrm{a}$.

${ }^{9}$ Heidegger, M., Prolegómenos para una historia del concepto de tiempo, p. 245.
} 
mundo del trabajo. Los entes, antes comprendidos en tanto artefactos, ahora se muestran ante la mirada contemplativa como cosas naturales. Sin embargo, cabe preguntar: ¿existen formas de comparecencia de la naturaleza dentro del contexto ocupacional del mundo del trabajo, es decir, experiencias de lo natural que no impliquen una desmundanización? ¿Cómo es la experiencia de la naturaleza que se ofrece a partir del contacto con los artefactos? 10

Todas las experiencias técnicas de lo natural coinciden en que la naturaleza se hace presente en tanto relacionada con la praxis, y no como algo ante los ojos, ofrecido a una experiencia contemplativa o tematizante. En estas experiencias, la naturaleza es descubierta como fenómeno accidental del trato ocupacional con artefactos, es decir, ella misma nunca comparece como ente a la mano, sino como algo en relación al útil y a la ocupación técnica; en otras palabras, aparece de manera concomitante e inexplícita con el trato pragmático. En El concepto de tiempo puede encontrarse una síntesis de los diversos modos de aparición técnica de la naturaleza. ${ }^{11}$ Allí se distingue la naturaleza como aquello con lo que cuenta la ocupación, aquello ante lo que se protege, y aquello a partir de lo que se produce algo. Observemos por separado estos fenómenos -y otros no explicitados:

${ }^{10}$ El concepto de naturaleza en Heidegger es problemático y ha dado lugar a muy numerosas interpretaciones, tanto del propio autor como por parte de sus comentadores. En esta oportunidad, optamos por circunscribir el problema de la naturaleza a aquellos aspectos de este fenómeno que hagan su aparición en el contexto de la operación técnica en el mundo -y esto, dentro de las obras del período de Marburgo. Así, quedarán excluidas del presente trabajo cuestiones tales como la naturaleza como lo inentendible, o lo inexplicable por antonomasia (Heidegger, M., Prolegómenos para una historia del concepto de tiempo, p. 273); naturaleza como caso límite del ente intramundano (Heidegger, M., Sery Tiempo, trad. de Jorge Eduardo Rivera, Madrid, Trotta, 2006, p. 114); naturaleza en tanto conjunto total de legalidades -sentido "kantiano"- (Heidegger, M., Lógica: la pregunta por la verdad, p. 250); naturaleza como physis, lo que crece y lo que ha crecido en aquel crecimiento (Heidegger, M., Los conceptos fundamentales de la metafísica. Mundo, finitud, soledad, trad. de Alberto Ciria, Madrid, Alianza, 2010, p. 59).

${ }^{11}$ Heidegger, M., El concepto de tiempo, trad. de Jesús Adrián Escudero, Barcelona, Herder, 2008, pp. 32-33. 
a) En primer lugar, la naturaleza viene a la presencia en el material (o el "de qué" [Woraus]) del ente técnico. ${ }^{12}$ Así, el proceso pragmático de martillar descubre el aspecto utilitario de los elementos de los que está fabricado este útil: la tarea que el martillo debe realizar demanda el peso y la solidez de la cabeza, además de la resistencia y comodidad del mango, características que remiten, a su vez, a su composición de madera y acero respectivamente, o bien otros elementos que satisfagan esas mismas necesidades. Este modo de concebir la naturaleza como materia también es estudiada en la lección Los problemas fundamentales de la fenomenología. En este escrito, la naturaleza se presenta como lo siempre presente, que no necesita ser producido, y, en este sentido, se ubica en la misma región entitativa que la materia, es decir aquello que la producción toma como base, pero que, a su vez, no ha sido elaborado. Sin embargo, en esta lección se observa una interesante aclaración, que será retomada por nosotros en un punto ulterior de nuestro análisis: allí se indica que los materiales "no son producidos totalmente". ${ }^{13}$ La adición de este adverbio indica que, en efecto, la materia de la que se sirve la tarea productiva ya se encuentra, en numerosas oportunidades producida, o como diremos más adelante, industrializada. Heidegger no desarrolla su afirmación, y el carácter ya previamente manufacturado de la materia queda sin tematizar.

b) Un segundo aspecto que dirige y da sentido al uso técnico de herramientas es el así llamado "para-qué" [Wozu]. Junto con el "de-qué", el "para-qué", orienta el obrar hacia una finalidad, que siempre coincide con la obra terminada (a su vez, ella recibe su sentido del por mor de qué). El para-qué cumple un papel tan fundamental que puede ser identificado con la misma esencia del útil, en efecto, según la postura de Heidegger, que podría ser caracterizada como "intencionalista", ${ }^{14}$ en los entes técnicos, su quidditas coincide con su función. El para-qué es capaz de traer la naturaleza a la presencia de un modo peculiar. El para-qué se encuentra íntimamente asociado al de-qué (materia prima), puesto que las características de la tarea que se desea

\footnotetext{
12 Heidegger, M., Ser y Tiempo, p. 98.

${ }^{13}$ Heidegger, M., Los problemas fundamentales de la fenomenología, trad. de Juan José García Morro, Madrid, Trotta, 2000, p. 151. Las cursivas son nuestras.

14 Parente, D. y Crelier, A., La naturaleza de los artefactos: intenciones y funciones en la cultura material, Buenos Aires, Prometeo, 2015, p. 20 y ss.
} 
realizar delinearán los aspectos que deberán estar presentes en la materia utilizada para que el ente técnico resultante sea exitoso. Una silla funcional no puede ser construida íntegramente de algodón. Según las características y comportamientos de los elementos utilizados (dureza, flexibilidad, resistencia, y también forma, tamaño, figura), la materia se revelará como adecuada o inadecuada para aquello que le otorga sentido a todo el proceso de producción: las finalidades propias del proyectar fáctico de la existencia. Por supuesto, todas aquellas características no son percibidas de manera teórico-temática, sino desde la comprensión pre-científica inherente a la circunspección que hace uso de las cosas. En cada uno de los mencionados materiales se acusa, como última referencia, la naturaleza, la cual, según se sugiere, ya no depende de ninguna instancia de producción. La naturaleza comparece aquí como lo disponible y adecuado, aquello sobre lo cual la ocupación puede extenderse para incorporarlo al plan técnico.

c) La naturaleza puede comprenderse como la misma posibilidad de obtención de materia prima y energía, es decir, la disponibilidad total de materiales en general. Este modo de visión descubre técnicamente a la naturaleza fijando su atención pre-teórica en las posibilidades inherentes al medio ambiente disponible. Heidegger afirma que la naturaleza puede resultar abierta en el mismo descubrimiento del mundo circundante. El río comparece como energía hidráulica, el bosque como reserva forestal, y el cerro como cantera. ${ }^{15}$ La utilización del paisaje para los fines técnicos de la ocupación revela a la naturaleza como reserva de energía y materia prima, que espera la intervención humana para poner sus posibilidades al servicio de la existencia. Este modo de aparición es quizás el más discutido por las críticas heideggerianas posteriores a la Kehre, que se centran en la concepción de la Gestell como esencia de la técnica contemporánea.

d) En cuarto lugar, y en un sentido quizás más omnipresente, pero, por lo mismo, más subliminal, puede destacarse el descubrimiento de la naturaleza como aquello en vistas a lo cual los objetos técnicos adquieren su configuración específica. La naturaleza está presente en el sistema de alumbrado público, en tanto que éste tiene en cuenta la oscuridad; el reloj lleva consigo la originaria referencia a una determinada posición del sol en el firmamento; el desagüe y el

${ }^{15}$ Heidegger, M., Sery Tiempo, p. 98. 
techo a dos aguas esperan la lluvia, a la vez que protegen de la intemperie. ${ }^{16} \mathrm{La}$ misma configuración de nuestro mundo circundante civilizado ha ido adquiriendo su forma actual en referencia a los fenómenos naturales de los cuales ha intentado protegerse o separarse de manera insistente y constante. En este sentido, podría afirmarse que la naturaleza comparece permanentemente en nuestra experiencia del mundo técnico [Werkwelt], pero en tanto que cancelada por éste, o, más bien, prevista en el diseño que persigue la protección contra ella. En este mismo sentido, en Los problemas fundamentales de la fenomenología también se presenta la naturaleza como "lo que opone resistencia a la transformación productiva". ${ }^{17}$ Esta interpretación se volverá muy relevante cuando, más adelante, estudiemos las limitaciones naturales a los procesos productivos. La consideración de la naturaleza como "lo previsto" en la producción abre las puertas para la aparición de un factor fundamental, cuyas implicancias en la configuración del mundo técnico reciben escasa elaboración; nos referimos a la noción de mundo público, desde un punto de vista técnicoproductivo. Esta noción será expandida en la segunda sección de nuestro trabajo.

Así pues, particularmente en el mundo-en-torno público, hay remisiones constitutivas que remiten a algo que siempre ya está abí, enseñándolo y descubriéndolo en cuanto lo que está ahí, en todo momento disponible sin que sea nada que se maneje expresamente en la ocupación ni que esté cortado a la medida de un Dasein individual y determinado, sino que cualquiera lo utiliza, y de la misma manera que cualquier otro. ${ }^{18}$

De lo analizado hasta este punto puede extraerse la siguiente conclusión: la naturaleza, lejos de su sentido científico y contemplativo, comparece de diversas maneras dentro del contexto pragmático de la ocupación, es decir, la naturaleza con sus procesos y causalidades, y con variado grado de explicitud, hace su aparición en medio del mundo circundante sin necesidad de que el Dasein suspenda su trabajo para considerarla. La naturaleza aparece, según se

\footnotetext{
${ }^{16}$ Heidegger, M., Prolegómenos para una bistoria del concepto de tiempo, p. 249.

${ }^{17}$ Heidegger, M., Los problemas fundamentales de la fenomenología, p. 151.

${ }^{18}$ Heidegger, M., Prolegómenos para una historia del concepto de tiempo, p. 249.
} 
indica, en el mundo-en-torno público. Esto significa que el Umwelt ya está desde siempre atravesado por, al menos, dos factores que exceden a la mera interrelación de funciones de los útiles, a saber, por la naturaleza -en tanto lo tenido en cuenta en el diseño y producción, en tanto disponibilidad de energías y en tanto materia prima- y por los otros, en tanto productores, consumidores, organizadores o destinatarios de las actividades técnicas. Heidegger indica que los artefactos obtienen su sentido de una totalidad de artefactos, cuyo modelo es el taller, y cuyo centro es el Dasein en el sentido de Worumwillen. Ahora bien, tomando en consideración la aparición de la naturaleza y de los otros en medio del taller, ¿no habría que expandir consecuentemente los límites del mundo circundante? ¿No será hora de abandonar el taller como modelo del Umwelt, y la técnica artesanal como modelo de operación técnica?

\section{Segunda parte: del werkwelt al conjunto técnico}

En nuestro estudio de los límites del mundo circundante técnico y su relación con las causalidades naturales y la actividad de los otros, hemos observado un problema: Heidegger reconoce la aparición de la naturaleza en el contexto de la ocupación pero le concede, o bien un papel completamente pasivo (usar la materia para tal o cual fin y/u obtener de ella energía de manera irrestricta), o bien la interpreta como algo ante lo cual sólo es posible responder de manera combativa o reactiva, por ejemplo: la oscuridad, el frío, la lluvia. Es decir, Heidegger reconoce que la naturaleza hace su aparición en el ámbito de la ocupación, pero, o bien la comprime fuertemente, presentándola como plástica y abierta a transformación (ignorando las exigencias que los fenómenos físicos aportan a la tarea productiva), ${ }^{19}$ o la expande exageradamente como fuerza natural ante la cual sólo cabe la defensa y protección.

Consideramos que la esfera de despliegue de la ocupación humana que Heidegger presenta se articula del siguiente modo simplista: existe una serie de artefactos, definidos por una finalidad, tal que cada uno de ellos se refiere, con diversos grados de explicitud, a otros, y últimamente, todos se muestran arraigados en una totalidad respeccional, un área de interés técnico que él

${ }^{19}$ Pensemos, por ejemplo, en la segunda ley de la termodinámica. 
denomina mundo circundante, o, en sentido técnico, mundo del trabajo. El fundamento último de este entramado es el proyectar (siempre público e histórico) del Dasein. De aquí puede concluirse que el centro de emanación y de configuración de sentido del mundo circundante es el ser humano, un ente que se despliega técnicamente en el mundo.

Si analizamos las diversas exposiciones del mundo circundante realizadas por el primer Heidegger, rápidamente comprenderemos que el modelo que el autor tiene en mente es el del taller, y no precisamente el de un taller mecánico o un taller de metalurgia, de electrónica, o de fotomecánica, sino un taller perteneciente a una etapa pre-industrial del desarrollo técnico humano (muy probablemente se esté pensando específicamente en un taller de carpintería).

El modelo del espacio técnico pensado por Heidegger es obsoleto. Pero esta afirmación no constituye ninguna novedad; una y otra vez se ha indicado que el modelo del Heidegger de los años '20 para pensar la interacción del ser humano con artefactos y entramados artefactuales es la técnica artesanal. Sin dudas, la restricción del campo de análisis traerá limitaciones a las descripciones resultantes del mundo del trabajo. En todo caso, las limitaciones que este recorte de la realidad técnica trae a la filosofía de la tecnología son numerosas e importantes. Aquí nos dedicaremos únicamente a analizar el modo en el que la restricción del ámbito técnico restringe también la visión de la estructura el mundo del trabajo.

Como vimos al comienzo de este estudio, en numerosas oportunidades, Heidegger equipara la noción de naturaleza con la noción de materia prima. Pero ¿a qué materia se refiere? Manifiestamente, hace referencia a acero, hierro, mineral, piedra, madera, etc. ${ }^{20}$ Sin embargo cabe realizar la pregunta: ¿cuál es la verdadera forma de aparición de estos materiales en la experiencia del artesano (si aceptamos a este tipo de actor técnico como modelo)? ¿Se relaciona acaso el constructor con madera viva extraída de un árbol, o con arena de hierro recolectada por él mismo de una cantera? ¿realmente comparece la madera del árbol en el mango del martillo, o es esta una abstracción que no encaja con la experiencia cotidiana? Tal vez podría responderse que nada impide que un

${ }^{20}$ Heidegger, M., Sery Tiempo, p. 98. 
artesano sea él mismo responsable de la tala del árbol sobre el que luego trabajará, pero ¿cuántas veces puede verificarse esta relación de extracciónproducción en la realidad técnica contemporánea? $\mathrm{Si}$ analizamos más minuciosamente los ámbitos técnicos post-industriales (a los cuales Heidegger ya pertenecía, pero no tematizó profundamente), pronto descubriremos que el artesano difícilmente se relaciona con el árbol viviente, implantado en el bosque, sino con tablones procesados por terceros, quienes, a su vez, se relacionaron con los árboles por medio de complejas maquinarias de procesamiento forestal.

La materia con la que el artesano se relaciona, aunque Heidegger diga ver en ella la naturaleza en tanto materia prima, es ya, ella misma, materia industrializada. ${ }^{21} \mathrm{El}$ caso del artesano que recolecta su propia materia es tan difícilmente verificable, que sería imprudente tomarlo como modelo para extraer consecuencias acerca de las características del mundo que rodea a ese tipo de operador técnico. La concepción de un artesano responsable de su materia ya no responde a la experiencia cotidiana de relación técnica con el mundo. La experiencia técnica ya se encuentra desde su comienzo atravesada por entramados mayores en los que se interconectan redes de extracción y procesamiento de materiales. El artesano siempre llega tarde a la operación sobre su materia; ésta ya ha sido previamente manipulada.

Pero tal vez Heidegger no quiera decir que la naturaleza aparezca como tal (en tanto materia) en el trabajo del artesano, entonces, ¿cómo podría aparecer? ¿'Tal vez en el segundo sentido que indicamos, a saber, como conjunto global de energías, o como lo permanentemente disponible para explotación? Si es así, el mundo circundante en tanto totalidad respeccional y conjunto de artefactos debe ser repensado, dado que sus límites se desdibujarían. En efecto, si en el simple martillo duerme la naturaleza, y la naturaleza no es sólo la materia de la que algo está hecho, entonces en el martillo también duerme una compleja red de extracción de energías; pero no sólo eso (y en este punto amplificamos la visión de Heidegger) sino también sistemas de distribución de bienes y maquinarias, de trabajos y sistemas accesorios, como la red de energía eléctrica, o el sistema de salud que posibilita el trabajo de los obreros, o incluso el sistema

${ }^{21}$ Este hecho se esconde detrás del adverbio "totalmente", que observamos hace un momento en el fragmento extraído de Los Problemas Fundamentales de la Fenomenología. 
educativo que los prepara, y el sistema de transporte que los traslada desde y hacia la mina o el aserradero. Si esto es así, en el martillo no sólo duerme la naturaleza como materia, tampoco únicamente como posibilidad de extracción de energías ni como disponibilidad total de los entes que están ahí, sino como algo más complejo que Heidegger no detectó: en el artefacto duerme lo que Simondon dio en llamar conjunto técnico.

Un conjunto técnico podría ser definido como una "soldadura de zonas funcionales". ${ }^{22}$ Esta noción hace referencia al ensamblaje no únicamente de máquinas y artefactos en un único ámbito que interrelaciona sus funcionamientos y los vuelve solidarios unos con otros, sino que se expande a relaciones funcionales de segundo grado tales como el sistema de distribución de materia prima, y el sistema social y económico de organización del trabajo y el descanso de los operarios y diseñadores. En este sentido se afirma que "no sería exagerado decir que la cualidad de una simple aguja expresa el grado de perfección de la industria de una nación". ${ }^{23}$

La versión más simple del conjunto técnico es aquella que sólo facilita la aproximación de operaciones y causalidades que de por sí solas no llegarían a interconectarse, el ejemplo de este modo del conjunto técnico es el laboratorio. ${ }^{24} \mathrm{El}$ mismo tiene, según Simondon, "un rol funcional de segundo grado": 25 no genera de primera mano las reacciones químicas que se buscan, pero aproxima los sistemas e individuos técnicos que deben interrelacionarse para que cierta reacción química tenga lugar. Ahora bien, para captar el sentido más amplio y complejo de conjunto técnico (el que mencionamos en primer

\footnotetext{
22 Simondon, G., El modo de existencia de los objetos técnicos, trad. de Margarita Martínez y Pablo Rodríguez, Buenos Aires, Prometeo, 2007, p. 93.

23 Ibidem.

24 Nótese la diferencia con el modelo de Heidegger. El laboratorio, a diferencia del taller de carpintería, implica un entrecruzamiento de procesos y causalidades, de máquinas y útiles de procedencias muy diversas, y surgidos de una etapa de invención que requiere de un cierto conocimiento teórico. Los individuos técnicos del laboratorio son, en efecto, objetos técnicos de elevada complejidad, esto es, objetos técnicos científicos. El ejemplo de Simondon no hace abstracción de las causalidades y subsistemas interrelacionados, sino que se coloca en el centro de esta problemática.

25 Ibidem, p. 84.
} 
lugar) es necesario "experimentar un modo de existencia". ${ }^{26}$ En efecto, la interrelación de sistemas, funciones, causalidades, regulaciones, usuarios y servicios auxiliares sólo aparece ante una mirada que atiende al mundo circundante tal como queda expandido por causa de la toma en consideración de las propiedades de la materia, la disponibilidad de energía y la aparición de otros seres humanos, en tanto mundo público. Esto significa que Simondon busca la estructura de la realidad técnica expandida en el mismo lugar que Heidegger ( $y$ por eso sus pensamientos se vuelven compatibles), a saber, en el análisis de la existencia: "el conjunto técnico sólo puede ser captado por intuición, porque no se deja considerar como un objeto aislado, abstracto, manipulable, a disposición del hombre. Corresponde a una prueba de existencia y de puesta en situación; está vinculada con la acción recíproca con el sujeto". 27

El autor francés es consciente de la íntima relación del conjunto técnico con el mundo y por eso indica que "el conjunto se mantiene en una medida, siempre inherente al mundo". ${ }^{28}$ De hecho, el conjunto se encuentra mucho más arraigado al medio circundante que una máquina o una herramienta (una represa sólo puede ser construida en el curso de un río, y una vez establecida, no puede ser simplemente trasladada).

En el conjunto técnico están implicados numerosos factores que exceden a la mera estructura remisional del taller. Ciertamente, Heidegger reconoce que dentro del contexto de la ocupación hace su aparición algo más que la naturaleza como energía disponible, o como materia prima. Dice que en el mundo del trabajo también comparece el portador o usuario, como lo tenido en cuenta a la hora de construir el artefacto; esta aclaración daría cuenta de la fase de diseño y antropometría necesaria para la producción de un objeto técnico. Con todo, hasta este punto, en el esquema heideggeriano el ser humano sigue apareciendo constreñido a los límites de una producción cuasi-privada en la que un productor ofrece un útil a un usuario por una vía limpia de encrucijadas.

\footnotetext{
${ }^{26}$ Ibidem, p. 243.

27 Ibidem, p. 244.

${ }^{28}$ Ibidem, p. 192.
} 
Sin embargo, Heidegger realiza una observación que amplifica la noción de lo circundante precisamente en el sentido que aquí nos interesa ${ }^{29}$. Dice:

junto con ello [lo producido] comparece el mundo en el que viven los portadores y consumidores, mundo que es también el nuestro. La obra de la que nos ocupamos en cada caso no está solamente a la mano en el mundo privado, por ejemplo, en el lugar de trabajo, sino que lo está en el mundo público. Con el mundo público queda descubierta y accesible a cada cual la naturaleza del mundo circundante. En los caminos, carreteras, puentes y edificios la ocupación descubre la naturaleza en determinada dirección. Un andén techado tiene en cuenta el mal tiempo; las instalaciones del alumbrado público, la oscuridad. ${ }^{30}$

El máximo grado de amplificación que el mundo circundante alcanza en el período de Marburgo es el que acabamos de revisar: el así llamado mundo público. Parece que ese mundo público (presentado en este fragmento en clave técnica, pero íntimamente relacionado con el Uno como estado público de interpretación) sólo nombra la disponibilidad social de los productos de construcción y diseño, así parecen indicarlo los ejemplos que se enuncian inmediatamente: caminos, carreteras, puentes, edificios, andenes techados.

En efecto, para Heidegger, así como en el útil está presente la naturaleza como materia prima y como factor ante el cual el diseño reacciona, también están presentes los otros seres humanos, según él dice, como portadores y consumidores. El mundo público parece mentar un ámbito compuesto por objetos técnicos destinados a todos, o bien, producidos para muchos. El mundo técnico público estaría poblado por artefactos de grandes dimensiones, construidos teniendo en cuenta una multiplicidad de usuarios posibles, y a ninguno en particular (una vez más, se observa el imperio del Uno). El mundo público estaría hecho de artefactos públicos. La privacidad del taller se rompe,

\footnotetext{
${ }^{29}$ Aunque no extrae las consecuencias de su propia afirmación: estas consecuencias se condensarían en la conversión de la noción de mundo del trabajo en el concepto más abarcativo de "conjunto técnico".

${ }^{30}$ Heidegger, M., Sery Tiempo, p. 98.
} 
pero no por la interconexión de causalidades, servicios o intereses políticos e ideológicos, sino tan sólo porque los artefactos, unilateralmente y estandarizadamente producidos, tienen múltiples destinatarios y múltiples productores. La función de estos artefactos sigue siendo explicada en los términos artesanales de producción con finalidad, o incluso más, aplicación de forma a materia. ${ }^{31}$

El mundo circundante conserva su carácter simplista, aunque se multipliquen los usuarios y productores. Aún puede ser explicado con los conceptos fundamentales de materia, producción, funcionalidad, intención humana, y naturaleza (como ante qué del construir). En definitiva, el mundo técnico público de Heidegger no es más que un taller amplificado. En esta estructura de conceptos fundamentales (materia, finalidad, productores, usuarios) que darían razón de toda la realidad técnica (puesto que no contamos con otros conceptos), nada se dice de la interconexión de industrias, de la tercerización de las tareas o de la presuposición de sistemas secundarios de mantenimiento, distribución, limpieza, o el casi omnipresente sistema de abastecimiento energético.

Si se pretende incluir el "aspecto humano" a la hora de considerar la dimensión mundana de la producción técnica, las figuras de productor y destinatario resultan insuficientes. Los seres humanos del proceso técnico no aparecen en las únicas modalidades de "productores" y "usuarios". También existen los responsables de mantenimiento y reparación, comerciantes, distribuidores, limpiadores, inspectores de salubridad... Pareciera que Heidegger condensa toda la multiplicidad y variedad de factores de la realidad técnica en la única figura omnipotente y ubicua del productor-artesano. Esta concepción está muy lejos de la situación técnica actual (e incluso lejos de la situación de los años '20, cuando Heidegger enseña las lecciones que aquí analizamos). Pero, podría preguntarse nuevamente, ¿no habla Heidegger acaso de complejos entramados industriales, como centrales hidroeléctricas y canteras? Si revisamos las secciones de su obra de los años '20 donde estos conjuntos industriales son nombrados, observaremos que los mismos son, justamente, sólo mencionados. Nunca se discurre sobre la complejidad de dichos sistemas. Pareciera que es de común conocimiento todo lo que sucede

${ }^{31}$ Simondon, G., El modo de existencia de los objetos técnicos, p. 258. 
en el interior de una represa, o de una mina de carbón, lo cual, manifiestamente, no es el caso. Incluso estos grandes sistemas son tratados en los términos simplistas del modelo artesanal: una naturaleza que hace su aparición como materia o disponibilidad de energía, un complejo artefactual transparentemente definido en su funcionalidad, que es interpuesto al recurso natural para extraer la energía, y un conjunto de operadores que, por medio de actividades misteriosas, nunca mencionadas, y que ocurren en el interior de ese sistema que es, a la vez, obvio en su funcionalidad, pero desconocido en su funcionamiento, consiguen el objetivo propuesto. Dada la ausencia de desarrollo de la descripción de los ejemplos propuestos, dada la falta de mención de cuál es la variación cualitativa que diferencia el taller y la fábrica, deberemos asumir que ambos se pliegan al mismo modelo.

Es cierto que Heidegger reconoce que en el artefacto, además de la naturaleza (como materia y como disponibilidad de energía), hacen también su aparición los otros. El problema radica en que no estudió las consecuencias de la inclusión del mundo público en el contexto del taller, es decir, no desarrolló la estructura de lo público en tanto "lo público técnico", o, dicho de otra manera, abandonó el problema en el momento en que el mismo se volvía político. Para nosotros, en cambio, la consecuencia de la aparición del mundo público en el contexto de la esfera ocupacional es la expansión del mundo circundante, una expansión que puede ampliarlo hasta volverlo irreconocible para los sencillos ojos del modelo artesanal. Un mundo prolongado de esta forma, para que incluya el universo de tareas anexas y concomitantes, y que reconozca la tercerización de un sinnúmero de actividades indispensables para la producción, se ampliaría hasta llegar a coincidir con la realidad planetaria total en tanto universo tecnificado, esto es, en tanto conjunto técnico o tecnósfera. ${ }^{32}$

${ }^{32}$ Nos servimos del término acuñado por el geólogo Peter Haff, que originalmente refiere a "la suma de la producción [output] material de la ocupación humana contemporánea (medida en toneladas)" (Haff, P., "Scale and diversity of the physical technosphere: a geological perspective", en The Anthropocene Review, Ed. Sage, 2016, p. 1; disponible en anr.sagepub.com.), pero nos permitimos amplificar su sentido para que también incluya -en nuestra interpretación- todo lo que no puede ser calculado en términos materiales de masa, pero que forma parte inseparable de la actividad productiva, como empleos, regulaciones, modos de organización, redes de 
$\mathrm{Al}$ incluir el mundo público en la descripción del artefacto que habita el taller, Heidegger abre las puertas para el ingreso de una realidad técnica mucho más compleja, más contemporánea, más semejante a nuestra experiencia cotidiana, pero no extrae las consecuencias de dicha inclusión. En este punto cobrará una importancia fundamental la filosofía de la técnica de Simondon. En efecto, si en el taller entran los otros, ingresarán con sus máquinas, con sus políticas, normativas, ideologías, modelos de eficiencia y productividad, circuitos de distribución y comercialización y sistemas asociados de salubridad, educación, vivienda y recreación, etc. La técnica que presenta el primer Heidegger no sólo no corresponde a un modelo contemporáneo, ni siquiera corresponde adecuadamente a un modelo tradicional: la figura del operario autosuficiente que es, además, artesano, recolector, productor y consumidor ha dejado de ser el prioritario desde hace más de mil quinientos años, o, en el mejor de los casos sólo se mantiene en sociedades aisladas, aldeas perdidas en medio de las montañas, o en las chozas de los ermitaños, que cortan ellos mismos la madera que tallan, y cosechan los vegetales que consumen, sin servirse de ningún subsistema técnico de mantenimiento o abastecimiento. En los demás casos, incluso las actividades técnicas artesanales se encuentran atravesadas por innumerables subsistemas y suprasistemas de interdependencias técnicas. El hierro del herrero es proporcionado por una serie de agentes que desconocen la herrería.

Nuestra propuesta es que, al estudiar los límites del mundo circundante, Heidegger llega a las orillas de un descubrimiento que hubiese vuelto su filosofía de la técnica (en tanto ontología de los objetos técnicos) mucho más adecuada al modo de funcionamiento actual de la misma. Cuando indica que en el artefacto (y por ello, en el mundo circundante) hacen su aparición la naturaleza como materia y energía, y los otros en tanto mundo público, el mundo circundante debería haber roto sus límites para emparejarse a semejante descubrimiento, en lugar de mantener forzadamente el modelo simplista de la producción artesanal, y, reconociendo que incluso el taller privado ya se encuentra industrializado, expandirse a un entramado técnico que considerara sistemas, subsistemas e infraestructuras de muy variadas características. La

comercialización, y factores simbólicos e históricos que orientan la producción de objetos técnicos en uno u otro sentido. 
caída del modelo del taller abre las puertas al modelo del conjunto técnico de Simondon.

Es cierto que los límites del mundo circundante llegan hasta donde se derrama la atención y el interés interpretativo y ocupacional del Dasein, ${ }^{33}$ pero en las obras de los años '20 sólo se estudiaron las configuraciones de un mundo circundante restringido. Nada se dijo de los límites de un Umwelt cuyo interés rector emana de un Dasein -que ya siempre, de hecho, se encuentracomprometido ocupacionalmente en sentido pluri-técnico y pluri-sistémico.

El mundo del trabajo sólo puede conservar su carácter acogedor, y hogareño, si uno se entrega sin ningún tipo de consideración a la tarea productiva. En el momento en que uno descubre algo más en el útil, como la naturaleza, el entramado remisional, o, particularmente, a los otros y el mundo público, los ojos circunspectivos se amplían y las múltiples fases del mundo técnico empiezan a hacer su aparición. De esta manera, el Umwelt de la cotidianidad estalla, y rápidamente adquiere dimensiones planetarias. El Umwelt, si es considerado profundamente, se convierte en conjunto técnico.

Realizamos una consideración final para concluir con la presente sección: Heidegger se refiere en el parágrafo 16 de Sery Tiempo a las así llamadas "formas deficientes de la ocupación". Éstas nombran experiencias que acontecen en medio del contexto pragmático del taller y durante el uso de artefactos, que fuerzan la atención del Dasein a captar (de manera pre-teórica) la estructura del mundo en tanto entramado remisional. Tales experiencias son la obstaculización de una tarea, la ruptura de un útil, y la desaparición de una herramienta. En ellas se vuelve manifiesto (pero no aún tematizado) el modo en el que un artefacto estaba interconectado con otros en un entramado remisional, y cómo el Dasein se ubicaba en el centro de esta estructura reticular que constituye el mundo. Pues bien, sugerimos que existen otras experiencias de deficiencia de la ocupación que aparecen si se disuelven los límites microtécnicos del mundo circundante hogareño. En efecto, en medio del trabajo con artefactos, puede ocurrir un corte de luz, puede suceder que no llegue a tiempo el reparto de madera, que la basura no sea recogida, o que se averíen los extractores o aparatos de climatización, o bien -para hacer nuestro punto más

${ }^{33}$ Heidegger, M., Sery Tiempo, p. 99. 
manifiesto- puede ocurrir una huelga de distribuidores. Todos estos problemas también nombran formas deficientes de la ocupación, pero no ya los de una ocupación aislada en el taller, sino integrada a un mundo técnico complejo y más realista. Así como la ruptura de un martillo es capaz de revelar la estructura reticular del mundo, una huelga de distribuidores es capaz de revelar la estructura inter-sistemática y técnica del mundo, esta vez amplificado a su dimensión de conjunto técnico o tecnósfera.

\section{Tercera parte: las exigencias de la materia}

En la sección anterior dijimos que, si se toman en consideración las realidades que se encuentran implicadas y latentes en el artefacto y en el taller (en tanto conjunto de útiles, o zengganz̧heit), rápidamente harán su aparición una serie de factores que, de ser tenidos en cuenta consecuentemente, llevarían a una expansión del mundo circundante. Dijimos que los factores que ingresarían a la realidad técnica serían: la materia (o el de-qué del artefacto y la obra), la naturaleza como disponibilidad de energías, y los otros en tanto mundo público de productores y consumidores. Ahora bien, existe un factor fundamental que también debería ingresar en la consideración del mundo del trabajo, un factor que ya se encuentra implicado en la comparecencia técnica de la naturaleza: las causalidades y procesos naturales que operan en el artefacto y que posibilitan, determinan y limitan su funcionamiento. La atención a estos factores causales llevará, por otra vía, a la disolución de los límites del mundo circundante artesanal. El propio Heidegger deja abierta esta posibilidad -aunque no la desarrolla- en la lección Lógica: la pregunta por la verdad. Allí se afirma:

[La naturaleza] es accesible en diversos modos. En primer lugar, de tal modo que, por así decirlo, en la captación vayamos a través de los utensilios que en primer lugar están dados previamente en el mundo circundante. Por ejemplo, levantando una silla y soltándola, puedo observar en ella el caer, y en concreto, esta silla no cae en la medida en que es silla sino en la medida en que está hecha de madera. En la medida en que la capto como naturaleza tengo que prescindir y prescindo de ella en la medida en que es silla, en atención a este modo de ser del caer, la silla no se diferencia del bastón o del sombrero, o de cualesquiera otros utensilios. Es decir, la naturaleza 
puede hacerse accesible por medio de un captar tal que prescinde del carácter primario de las cosas. Pero la naturaleza también puede experimentarse directa y precientíficamente cuando hablo de una cascada allí afuera en la naturaleza o de una piedra que se desprende de la roca y cae. Igual de originalmente que tengo la mesa, la silla y el sombrero, tengo la naturaleza en el sentido de "la naturaleza allí afuera".34

En este fragmento se observa una doble captación de lo natural: por un lado, como región de objetos accesible a una actitud tematizante y desmundanizante, pero, por otro lado, se presenta la naturaleza como un conjunto de procesos y causalidades operantes de antemano en los entes del mundo circundante. Esta es la captación llamada "directa y precientífica". Ahora bien, aunque no se explicita en el fragmento, es posible llegar la siguiente conclusión: si la silla cae y la piedra cae, y es posible tener una experiencia precientífica del caer de la piedra, también será posible tener una experiencia precientífica del caer de la silla. En la experiencia del caer de la silla ya se capta la naturaleza tan directa y precientíficamente como se capta la cascada o la caída de una piedra. Esta experiencia directa y precientífica de las uniformidades de procesos del mundo puede entenderse como una comprensión de habitualidad del "estilo global empírico" o "estilo causal universal"35 del mundo circundante.

El hacedor de espadas comprende que el acero cambia su color a medida que se lo calienta (aunque desconoce la ley de Rayleigh-Jeans, que predecía, hasta principios del siglo XX, la emisión de radiación térmica de los cuerpos). También comprende que ciertos colores (asociados a temperaturas) son los indicados para forjar y otros son los indicados para templar el metal. Incluso más, el herrero sabe que si se intenta templar el metal (sumergiéndolo en agua, por ejemplo) cuando el acero brilla en un tono inadecuado, el proceso podría finalizar en la ruptura de la espada. Resulta manifiesto que el operario comprende que ciertos procesos y encadenamientos fenoménicos duermen en

\footnotetext{
${ }^{34}$ Heidegger, M., Lógica: la pregunta por la verdad, p. 249.

${ }^{35}$ Husserl, E., La crisis de las ciencias europeas y la fenomenología trascendental, Buenos Aires, Prometeo, 2008, p. 73.
} 
la materia sobre la que él trabaja, y, para que su tarea sea exitosa debe adaptarse a dichos encadenamientos causales.

Lo que intentamos afirmar es que la actividad humana ya se encontraba delimitada por procesos inherentes a la materia, mucho antes de que estos fueran tematizados; en otras palabras, la comprensión primaria del quehacer productivo acarrea una comprensión primaria de los límites que los procesos latentes traen a ese quehacer. Estos límites le revelan al operario que el centro de la escena técnica no está ocupado por él, sino que su posición está nivelada con la de la materia y los artefactos y máquinas.

En un proceso posterior, y derivado, las regularidades del quehacer involucrado con la materia pueden ser formulados en forma de leyes científicas que, incluso luego de su derivación continuarán delimitando las actividades productivas humanas.

Ahora que el problema de los límites impuestos por los procesos naturales al quehacer artesanal ha sido enunciado, volvamos una vez más al taller para plantearnos el siguiente interrogante: ¿dónde se construyen los talleres? ¿Acaso en el interior de las casas, en medio de las alacenas, los sillones, las bibliotecas y las cocinas, o más bien en un espacio separado, y expresamente alejado del terreno en el que ocurre el descanso, la alimentación, y la higienización? ¿Es por mero capricho que, casi en la totalidad de los casos, las camas son expulsadas de los talleres? ¿O esta práctica responde a un ordenamiento inherente a la misma estructura de un taller? Resulta claro que el espacio técnico del taller trae consigo una serie de factores que resultan incompatibles con la actividad del descanso, aspectos que incluso podrían poner en riesgo la salud de quien buscase permanecer en su interior más tiempo que el necesario para completar los proyectos técnicos. Los talleres traen consigo ruidos, olores, desperdicios o niveles de temperatura que resultan aceptables para el que trabaja, pero inaceptables para el que descansa.

La instalación de las camas lejos de los talleres no es antojadiza. En el diseño del espacio del trabajo y el espacio del descanso están necesariamente previstas o tenidas en cuenta las características inherentes a cada actividad y las causalidades y procesos que en ellas se desarrollan. La cadena causal del acto de soldar dos metales necesariamente se encontraría en interferencia con la cadena causal del acto de dormir. El diseño y disposición del espacio técnico 
tiene en cuenta, aunque no se la detecte de manera expresa, una amplia y compleja serie de procedimientos, funcionamientos, actividades y causalidades que no son precisamente centrales al proceso de producción, pero, incluso en su carácter accesorio o concomitante, son suficientes para exigir un rediseño del espacio, en función de su manera de afectar el vivir y trabajar humanos. Ante esos factores, la actividad humana que ordena áreas significativas no puede más que adaptarse, o replantear la distribución del mundo circundante en función de una o varias causalidades intersecantes que provocarían un choque (incómodo en el mejor de los casos, catastrófico en el peor) de vectores pragmáticos incompatibles.

El espacio del trabajo y el del descanso no suelen convivir, y esto no ocurre únicamente por una serie de convenciones sociales e históricas, sino -y acaso principalmente- porque las operaciones que se realizan sobre la materia en el acto de producción vehiculizan o desencadenan un entramado de procesos y consecuencias que llevan a las dos tareas a distanciar sus encadenamientos, y en esa misma separación, a dividir físicamente las áreas en las que cada una de ellas será llevada a cabo.

Lo que aquí se intenta afirmar es que el mundo circundante expuesto y analizado por Heidegger en Sery Tiempo, puesto que el mismo queda asociado al modelo artesanal que no considera el problema de los conjuntos técnicos, resulta demasiado simple para dar cuenta de la complejidad de la realidad técnica contemporánea en términos espaciales y de interrelación pragmática de sistemas y causalidades. Los procesos, los artefactos, y los subsistemas también son capaces (y tal vez lo sean en primera medida) de definir los límites del Werkwelt. El mundo circundante no se abre solo en función del interés del Dasein, también se abre hasta donde lo permiten o lo exigen los materiales, las máquinas, las causalidades técnicas y las consecuencias implicadas en todas ellas.

En numerosas oportunidades el rol del agente humano es pasivo ante lo irrefrenable de un fenómeno físico que ocurre en el contexto de un medio técnico. Por ejemplo, un resonador magnético -si se desea evitar las interferencias con el equipamiento de medición- deberá ser instalado en el interior de una jaula de Faraday; un (ya antiguo) taller de revelado fotográfico sólo puede ser construido en un ambiente oscuro, o de muy baja luminosidad 
para proteger el material fotosensible; una fábrica de microprocesadores no tolerará el ingreso de polvo al espacio técnico, por lo cual la misma deberá tomar numerosas medidas de refrigeración y filtrado de aire, las cuales modificaran radicalmente la estructura edilicia.

Todas estas situaciones definen, con independencia de la conveniencia del Dasein, la forma, figura, tamaño, y localización del espacio técnico. Casi todos los espacios técnicos adquieren su configuración fáctica no porque el ser humano lo prefiera de tal o cual modo, sino porque de otra manera, los procesos físicos buscados no ocurrirían -o interferirían unos con otros- y la actividad técnica se detendría. Ilustremos nuestra posición por medio de algunos ejemplos:

1) Las torres de refrigeración de una planta nuclear tienen forma hiperboloide y alturas de hasta 200 metros puesto que esta forma y tamaño maximiza el efecto de succión o convección térmica (stack effect), proceso que posibilita el enfriamiento del agua caliente, resultado del sistema de enfriamiento del reactor. La forma de esta enorme edificación es la que más aprovecha un principio natural operante desde antes de la construcción de plantas nucleares, y desde antes de la existencia de seres humanos. 2) Las ventanas de los aviones no son redondeadas y pequeñas debido a una unilateral decisión de diseño de los constructores, sino debido a que ventanas más grandes y de bordes rectangulares son mucho más propensas a fisurarse en las esquinas ante la fuerza que ejerce sobre ellas la presurización de la cabina, y de desencadenar una explosión en mitad del vuelo (accidentes del Havilland Comet entre 1953 y 1954). 3) Los barcos que transitan del océano Atlántico al Pacífico (y viceversa) sin realizar la vuelta alrededor de América del Sur (ruta de Magallanes) no superan los 294,1 metros de largo por 32,3 de ancho, por 12 de profundidad; esto no ocurre porque los diseñadores de barcos hayan llegado a un arbitrario consenso en la construcción, sino porque aquellas medidas representan el tamaño máximo de embarcaciones admitidas por el canal de Panamá.

El aspecto que Heidegger no detectó (o no analizó) es que la materia y las causalidades naturales operantes en y entre las máquinas y artefactos traen sus propias exigencias al diseño del mundo circundante. Ciertas máquinas o artefactos exigen la construcción o conveniente disposición de otras máquinas 
y útiles (pensemos, por ejemplo, en la cama colocada debajo del ventilador de techo, o la consola de videojuegos colocada delante del televisor). Ciertos materiales exigen una disposición específica del espacio. Las máquinas también diseñan el mundo circundante (aunque en sentido impropio) Este Umwelt se expande, por un lado, hasta donde el interés del Dasein se despliega, pero por el otro, hasta donde la naturaleza (y diremos también, el carácter público, e histórico de la labor técnica) se lo permite. La noción de conjunto técnico amplifica la de Werkwelt porque tiene en cuenta los subsistemas y las causalidades, es decir, tiene en cuenta la voz de la materia, de la máquina y de la organización social que rodea y posibilita la producción.

Ahora bien, nuestra afirmación no debe ser malinterpretada: sin dudas, las máquinas no tienen capacidad de proyectar; ellas no poseen el modo de ser del Dasein, es decir, no existen, y por ello no tienen acceso a un universo de sentido. La estructura fundamental del mundo, la significatividad, sólo es accesible para un ente que es en el modo del existir, comprender y proyectar: "sólo el Dasein puede estar dotado de sentido o desprovisto de ép". 36 No decimos que los artefactos y sistemas técnicos tengan mundo, o acceso al fenómeno existencial del sentido, pero sí afirmamos que su modo de venir a la presencia trae una serie de exigencias ante las cuales el Dasein (punto donde acontece el sentido y la comprensión) sólo puede comportarse de manera adaptativa, reestructurando el mundo circundante de modo que ellas sean tomadas en consideración, de lo contrario, el Werkwelt se vendría abajo por entrecruzamientos de causalidades y procesos incompatibles. Será suficiente pensar en los trabajadores muertos o heridos durante la construcción del puente de Brooklyn: su trabajo de excavación se realizaba en enormes cajas de madera sumergidas (caissons) en el lecho del Río Este, sin tomar en consideración los problemas que los repentinos cambios de presurización, e, incluso más, el uso de explosivos dentro de ambientes presurizados, causaba en los operarios. Un Werkwelt que no considera las causalidades físicas acaba por colapsar, a veces, llevándose consigo al ser humano que trabaja en él.

Desde luego que sólo el Dasein configura mundo del trabajo, pero no puede hacerlo sin atender a las exigencias físicas, logísticas, históricas y sociales que las máquinas y los subsistemas le presentan de manera insistente. Una

${ }^{36}$ Heidegger, M., Ser y Tiempo, p. 175. 
perspectiva analítica del mundo circundante que considere estos aspectos necesariamente acabará por desmantelar los límites hogareños y artesanales del taller imaginado por Heidegger, y llevaría a una más precisa expansión del mundo circundante en un conjunto técnico.

\section{Conclusión}

Este trabajo fue realizado para mostrar el modo en que la insuficiencia del modelo del taller (y su consecuente noción de mundo circundante artesanal) puede ser completada por la noción de conjunto técnico de G. Simondon. Esta expansión fue sugerida por Heidegger, pero nunca fue llevada a sus consecuencias directas, es decir, la disolución de los límites del Umwelt artesanal y su conversión en una esfera técnica donde se interconectan aspectos, procesos, causalidades y fenómenos de carácter social e histórico, material, natural y causal, y por supuesto, semántico y pragmático. El mundo circundante expandido que Heidegger sólo delineó puede ser completado por la filosofía de la tecnología de G. Simondon.

\section{Referencias bibliograficas}

Heidegger, Martin, "La pregunta por la técnica", en Conferencias y artículos, traducción de Eustaquio Barjau, Barcelona, Ediciones del Serbal, 1994.

Heidegger, Martin, Los problemas fundamentales de la fenomenología, trad. de Juan José García Morro, Madrid, Trotta, 2000.

Heidegger, Martin, Lógica: la pregunta por la verdad, traducción de Alberto Ciria, Madrid, Alianza, 2004.

Heidegger, Martin, Ser y Tiempo, traducción y notas de Jorge Eduardo Rivera, Madrid, Trotta, 2006.

Heidegger, Martin, Prolegómenos para una historia del concepto de tiempo, traducción de Jaime Aspiunza, Madrid, Alianza, 2007.

Heidegger, Martin, El concepto de tiempo, traducción de Jesús Adrián Escudero, Barcelona, Herder, 2008. 
Heidegger, Martin, Los conceptos fundamentales de la metafísica. Mundo, finitud, soledad, traducción de Alberto Ciria, Madrid, Alianza, 2010.

Haff, Peter et al., "Scale and diversity of the physical technosphere: a geological perspective", en The Anthropocene Review, Ed. Sage, pp. 1-14; disponible en anr.sagepub.com.

Husserl, Edmund, La crisis de las ciencias europeas y la fenomenología trascendental, traducción de Julia V. Iribarne, Buenos Aires, Prometeo, 2008.

Parente, Diego, y Crelier, Andrés, La naturaleza de los artefactos: intenciones y funciones en la cultura material, Buenos Aires, Prometeo, 2015.

Simondon, Gilbert, El modo de existencia de los objetos técnicos, traducción de Margarita Martínez y Pablo Rodríguez, Buenos Aires, Prometeo, 2007.

Simondon, Gilbert, La individuación. A la luz de las nociones de forma y de información, traducción de Pablo Ires, Buenos Aires, Cactus, 2009.

Tugendhat, Ernst., Der W arheitsbegriff bei Husserl und Heidegger, Berlin: Walter de Gruyter \& Co., 1970

Recibido: 05/2018; aceptado: 10/2018. 